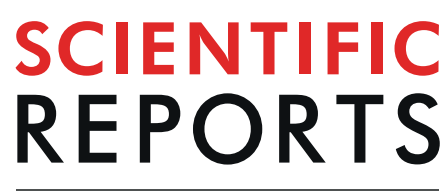

natureresearch

\title{
A new tool CovReport generates easy-to-understand sequencing coverage summary for diagnostic reports
}

\author{
Mark Gorokhov ${ }^{1}$, Mathieu Cerino ${ }^{2,3,4}$, Jérémie Mortreux ${ }^{2,3,5}$, Florence Riccardi ${ }^{2,3,5}$, \\ Nicolas Lévy ${ }^{2,3}$, Marc Bartoli $\mathbb{C}^{2}$, Martin Krahn ${ }^{2,3}$ \& Svetlana Gorokhova $\mathbb{1}^{2,3^{*}}$
}

In order to properly interpret the results of a diagnostic gene panel sequencing test, gene coverage needs to be taken into consideration. If coverage is too low, an additional re-sequencing test is needed to make sure that a pathogenic variant is not missed. To facilitate the interpretation of coverage data, we designed CovReport, a novel easy-to-use visualization tool. CovReport generates a concise coverage summary that allows one-glance assessment of the sequencing test performance. Both gene-level and exon-level coverage can be immediately appreciated and taken into consideration for further medical decisions. CovReport does not require complex installation and can thus be easily implemented in any diagnostic laboratory setting. A user-friendly interface generates a graphic summary of coverage that can be directly included in the diagnostic report. In addition to a stand-alone version, we also provide a command line version of CovReport that can be integrated into any bioinformatics pipeline. This flexible tool is now part of routine sequencing analysis at the Department of Medical Genetics at La Timone Hospital (Marseille, France). CovReport is available at http://jdotsoft.com/CovReport.php. It is implemented in Java and supported on Windows, Mac OSX and LinuX.

Since the arrival of short-read sequencing technologies, significant efforts have been spent to improve the quality of the obtained data in order to satisfy the requirements of molecular diagnostics. However, many regions of the human genome remain difficult to analyze using standard short-read sequencing approaches. These "dark" genome regions contain a number of genes responsible for human diseases ${ }^{1}$. For example, several neuromuscular disease-causing genes, such as NEBULIN (NEB) and SELENON (SEPN1), overlap these difficult to sequence regions. Disease-causing variants in gene regions with suboptimal sequence coverage can be overlooked. Thus, when short-read sequencing is performed in a diagnostic setting, identifying poorly-covered regions is critical for the interpretation of a diagnostic sequencing result. If coverage is not sufficient even for a small region of a highly suspected candidate gene, an additional re-sequencing test is needed to make sure that the pathogenic variant is not missed. To facilitate the coverage data interpretation by molecular geneticists and by test prescribers, we designed a novel easy-to-use visualization tool, CovReport. The concise coverage data summary generated by CovReport allows one-glance assessment of the sequencing test performance.

\section{Methods}

Implementation. CovReport is implemented as a standalone Java application. It can run on any platform where Java Runtime (JRE) is installed (Windows, Mac OS and Linux). The supported Java version is 8 . The application is using open source external libraries (JARs) which are embedded into the main executable JAR. The following external dependencies are used:

- Apache PdfBox (https://pdfbox.apache.org),

- Apache CLI (http://commons.apache.org/proper/commons-cli),

- JarClassLoader (http://www.jdotsoft.com/JarClassLoader.php).

${ }^{1}$ JDotSoft, Germantown, MD, USA. ${ }^{2}$ Aix Marseille University, INSERM, MMG, U1251, Marseille, France. ${ }^{3}$ Department of Medical Genetics, La Timone Hospital, AP-HM, Marseille, France. ${ }^{4}$ Laboratory of Biochemistry, La Conception Hospital, AP-HM, Marseille, France. ${ }^{5}$ These authors contributed equally: Jérémie Mortreux and Florence Riccardi.

*email: svetlana.gorokhova@univ-amu.fr 
The application could be downloaded from http://www.jdotsoft.com/CovReport.php as a compressed CovReport.zip file. After extraction into the local drive, the folder contains the following:

- ms 9 - folder with internalization message files used for the generated PDF file

- CovReport. jar - Java executable archive

- run. cmd - Windows command to start the application

- runFromCommandLine. cmd - Windows command line helper

After CovReport first execution the following items are created:

- pdf-results - folder with PDF files generated by the application

- CovReport. config - file with configuration persistence data; this file could be manually updated and reused to replace default for command line execution

The application can be started with user interface (UI) or from command line. The command line options are:

$-i,--i n p u t<$ arg $>$ input file

$-\mathrm{n},--$ name $<$ arg $>$ patient name

$-\mathrm{c},--$ config $<$ arg $>$ config file (optional)

The input file containing per-exon coverage information is a tab delimited CSV file. All entries in the file can be quoted per CSV file standard or can be without quotes. There should be no special characters in the input file. The following columns are expected: RefSeqName, GeneSymbol, Exon, Size, Mean Depth, SD Depth, Coverage $1 \times$, Coverage $5 \times$, Coverage $10 \times$, Coverage $20 \times$, Coverage $30 \times$. An example of an input file is provided in the Supplementary data (also available at http://www.jdotsoft.com/CovReport/44genes-stat_coverage_exon.txt). The input file can be easily generated by the Coverage Module of VarAFT tool ${ }^{2}$ (https://varaft.eu/) that uses BEDTools $^{3,4}$ to calculate exon-level coverage. However, results of any other coverage calculating pipeline can be converted into the appropriate format and used as CovReport input. The command line version of CovReport allows integrating this visualization tool as a step in an automated diagnostic sequence analysis pipeline.

The application user interface is in English, but the generated PDF file is in the current locale language. English and French are supported in the current distribution, with the possibility to add other languages by the user. The text included in the output pdf report can also be customized, making it possible to adopt CovReport in any diagnostic setting.

Detailed instructions for downloading and running CovReport are described at http://www.jdotsoft.com/ CovReport.php.

Exome sequencing of NA12878. DNA for the reference sample NA12878 was obtained from the Coriell Institute for Medical Research Repository (Coriell Institute, Camden, NJ, USA). Whole Exome Sequencing (WES) was performed by the Genomics and Bioinformatics Platform (GBiM) from the U 1251/Marseille Medical Genetics facility, using the NimbleGen SeqCap EZ MedExome kit (total design size $47 \mathrm{Mb}$ ) according to the manufacturer's protocol (Roche Sequencing Solutions, Madison, USA). The SeqCap EZ MedExome kit targets the entire human exome with enhanced coverage of exons from medically relevant genes in Mendelian diseases. Enriched fragment libraries were sequenced on the Illumina NextSeq 500 platform (Illumina, San Diego, CA, USA) using a $150 \mathrm{bp}$ paired-end sequencing protocol. Raw data were mapped to the built of the human genome (hg19) by using BWA 0.7.5.

\section{Results}

CovReport allows one-glance overview of exon coverage for a diagnostic sequencing test by generating a concise easy-to-understand report in the PDF format. Intuitive interface and absence of complex installation steps makes CovReport easy to apply by users without special bioinformatics training. CovReport can also be launched by command line allowing integration into any bioinformatics pipeline.

Several features make CovReport especially useful in a diagnostic setting. First, the application runs on a local computer allowing complete data security. Second, the patient's name from the previous analysis is automatically reset upon entering the new coverage file in order to avoid identity errors. Third, the format, content and the language of the report can be easily customized by the laboratory, adapting to any diagnostic setting. Fourth, information about additional Sanger re-sequencing of suboptimally covered regions can be integrated into the coverage report of the initial short-read sequencing allowing easy tracing of sequencing experiments.

Exon coverage for genes on the panel is visualized by drawing exons shaded according to the level of coverage: $100 \%$ covered exons are white, $90-100 \%$ exons are light gray, $<90 \%$ covered exons are dark gray, non-covered exons are black. Several visualization options allow adapting the graphical presentation to the user's needs. Genes with $100 \%$ coverage can be listed at the top of the report without drawing the exons (Skip white genes option, default). Schematic gene/exon structures will be drawn for the remaining genes, shading the lower-coverage exons. If Merge white exons option is selected, CovReport will fuse exons covered at $100 \%$ for more compact representation, which is useful for genes with numerous exons. Unchecking this option will produce the report with each individual exon drawn. Similarly, exons with the same shading can be merged for more compact representation (Merge non-white exons option). Average per-gene coverage can be shown next to each gene (Show gene weighted coverage option), which is also used for statistics of the total gene panel coverage (Show statistics option). RefSeq transcript information can be included in the report (Show gene transcripts option), recommended if transcripts 


\title{
Appendix 1. Coverage of target sequences
}

\author{
Patient name: NA12878 MedExome
}

The following genes are $100 \%$ covered (with a depth of at least 20x):

ANO5 (NM_213599), BVES (NM_147147), CAPN3 (NM_000070), CAV3 (NM_033337), COL6A1 (NM_001848),

COL6A2 (NM 001849), COL6A3 (NM 004369), DAG1 (NM 001165928), DES̄ (NM 001927), DNAJB̄6 (NM 058246), DPM3 (NM 153741), FHL1 (NM 001159702), FKRP (NM 024301), FKTN (NM 001079802), GAA (NM 000152),

GAA (NM_001079803), GMPPB (NM_013334), GNE (NM_001128227), HNRNPDDL (NM_001207000), HNRNPDL (NM_031372), LMNA (NM - 170707), MYOT (NM_006790), PLEC (NM_000445), POMGNT1 (NM_001243766), POMT1 (NM_007171),

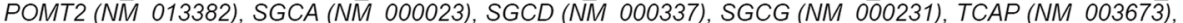
TNPO3 (NM_012470), TRAPPC11' (NM_021942), TRIM3̄2 (NM_012210), TTN $\overline{(N M}$ _133379)

Certain parts of the following genes are not $100 \%$ covered (see details below)

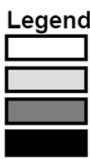

exons covered at $100 \%$ (with a depth of at least $20 x$ )

exons covered at $90-100 \%$ (with a depth of at least $20 x$ )

exons covered at $<90 \%$ (with a depth of at least $20 x$ )

exons not covered (with a depth of at least 20x)

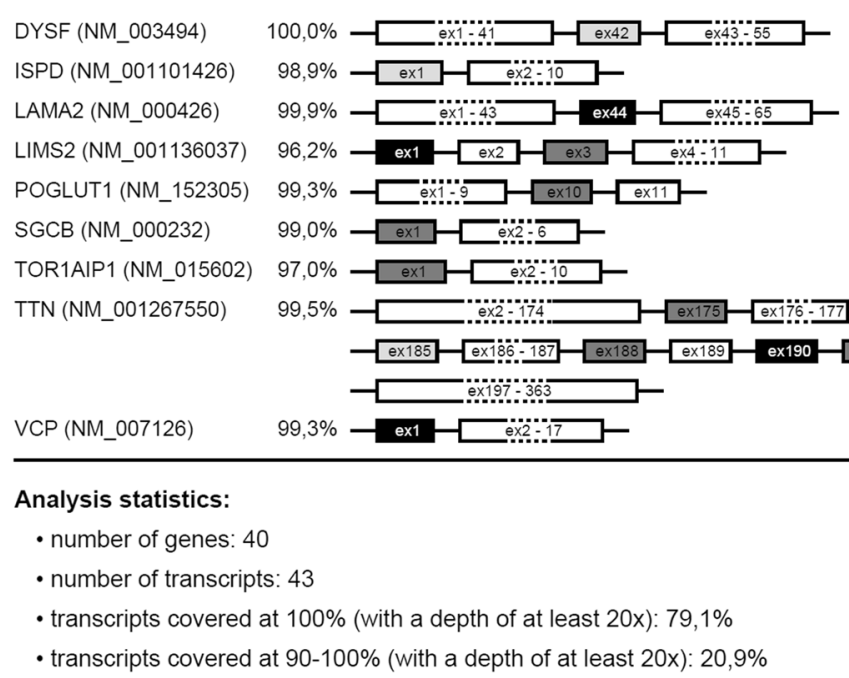

Analysis statistics:

- number of genes: 40

- number of transcripts: 43

- transcripts covered at $100 \%$ (with a depth of at least $20 x$ ): $79,1 \%$

- transcripts covered at $90-100 \%$ (with a depth of at least $20 x$ ): $20,9 \%$

Figure 1. Example of a coverage report generated by CovReport. An example report was generated by CovReport in order to visualize the sequencing coverage of 40 genes on LGMD (Limb Girdle Muscular Dystrophies) panel using exome sequencing data for the standard DNA sample NA12878. The name of the patient is listed as specified in the CovReport interface. In the top part of the report, the genes covered at 100\% are listed (if option "Skip white genes" is activated). Next, genes with sub-optimal coverage are shown with the exon shading according to the coverage level. The percentage of gene-level coverage is shown for each suboptimally covered gene (if option "Show gene weighted coverage" is activated). Finally, the coverage statistics for the genes analyzed is shown (if option "Show statistics" is activated).

differ in exon number leading to differences in coverage between isoforms. The default 20x depth of coverage in the report can be changed to $1 \times, 5 \times, 10 \times$ or $30 \times$. Finally, additional comments can be included in the report.

\section{Use case}

CovReport has been used for all diagnostic gene panel sequencing tests at the Laboratory of Molecular Genetics at the Timone Hospital (Marseille, France) since June 2018. The reports generated are directly annexed to the diagnostic results of various sequencing panels. Moreover, the coverage reports produced by CovReport are also routinely used for technical validation of the test since gene coverage statistics for the panel are integrated in the output.

To demonstrate the performance of CovReport, we used exome sequencing data for NA12878 to visualize the coverage of 40 genes on the extended Limb-Girdle Muscular Dystrophy (LGMD) gene list defined by the French National Network for Rare Neuromuscular Diseases ${ }^{5}$. Coverage module of VarAFT ${ }^{5}$ was used to obtain exon coverage for these genes. The default options were used to generate the report using CovReport with Show gene transcript and Show statistics options activated. As seen from Fig. 1, coverage was visualized for 43 transcripts corresponding to 40 genes. Most of the genes were covered at $100 \%$. The advantage of using Show gene transcript option is clear from this example, since TTN (NM_133379) is covered at 100\%, while TTN (NM_001267550) has several exons with lower coverage. Indeed, NM_133379 isoform is much shorter, as exons 50-219 of NM_001267550 are not included in this transcript. Six exons had no regions with above 20x threshold coverage (black exons). Several other exons had suboptimal coverage in regions corresponding to more than $10 \%$ of their length (dark gray). If similar coverage of these genes is obtained after a diagnostic sequencing for a patient affected with LGMD, the prescribing clinician will take into account the presentation of the disease in order to 
decide if the phenotype of the patient could be potentially explained by pathogenic variants in the exons with low coverage. If that is the case, additional resequencing of these regions will be necessary.

\section{Discussion}

The importance of coverage data is well established in genetic diagnostics. Indeed, most sequencing reports provide some information about coverage of target regions. However, these data are usually presented as an overall gene panel coverage or as an average-per-gene coverage. To our knowledge, no exon-level coverage data are provided with diagnostic reports. However, the distribution of pathogenic variants in a given gene is not homogeneous, as the disease-causing amino acid changes are often clustered in functionally important regions of the protein. Thus, information about coverage of the regions where disease-causing variants are concentrated is much more informative than an overall average gene coverage ${ }^{6}$. CovReport highlights individual exons with suboptimal coverage, facilitating the interpretation of the sequencing data.

The ultimate goal of diagnostic sequencing is to obtain an above-threshold coverage for the entire genomic area targeted by a given gene panel. This is feasible for small panels with genes that can be robustly sequenced. However, this goal is much harder to achieve for larger gene panels containing difficult to sequence genes. This is the case for the neuromuscular disorder field, since 33 out of 203 genes on the consensus myopathy gene lists ${ }^{5}$ contain "dark" regions of the genome that are not easily accessible using standard short-read sequencing approaches ${ }^{1}$. Disease-causing variants in these regions can therefore be overlooked leading to a false negative molecular diagnostic result. CovReport effectively highlights and draws attention to the exons with suboptimal coverage, allowing the molecular genetisist and the test prescriber to evaluate the need for a complementary re-sequencing test for these areas.

Since the arrival of high-throughput sequencing technology, several tools have been developed for calculating sequencing coverage, such as BEDTools genomecov ${ }^{3,4}$, Sambamba $^{7}$ and Mosdepth ${ }^{8}$. Diagnostic laboratories use one or combination of these tools as part of their standard sequencing analysis pipelines. However, the optimal way to visualize and evaluate the obtained coverage data is not universally defined. Several tools have been designed to evaluate the coverage of target regions after short-read sequencing in a diagnostic setting ${ }^{2,9,10}$. While these tools are useful to monitor quality control of runs and samples during technical validation of sequencing tests, unlike CovReport, they do not generate exon-level coverage visualization that can be directly annexed to the diagnostic report. To our knowledge, CovReport is the first tool that allows comprehensive and visual evaluation of coverage results for a sequencing test, allowing molecular geneticists or prescribing physicians to make important diagnostic decisions, such as pursuing further explorations of the poorly covered genomic regions.

In conclusion, CovReport generates a one-glance graphical overview of coverage for individual exons on a gene panel, facilitating interpretation of the sequencing test. CovReport is flexible and easy-to-use, making it easy to adopt in any diagnostic setting.

\section{Data availability}

CovReport is available at http://jdotsoft.com/CovReport.php.

Received: 4 December 2019; Accepted: 20 March 2020;

Published online: 10 April 2020

\section{References}

1. Ebbert, M. T. W. et al. Systematic analysis of dark and camouflaged genes reveals disease-relevant genes hiding in plain sight. Genome Biol. 20, 97 (2019).

2. Desvignes, J.-P. et al. VarAFT: a variant annotation and filtration system for human next generation sequencing data. Nucleic Acids Res. 46, W545-W553 (2018).

3. Quinlan, A. R. \& Hall, I. M. BEDTools: a flexible suite of utilities for comparing genomic features. Bioinformatics 26, 841-842 (2010).

4. Quinlan, A. R. BEDTools: The Swiss-Army Tool for Genome Feature Analysis. Current Protocols in Bioinformatics vol. 47 11.12.1-11.12.34 (2014).

5. Krahn, M. et al. A National French consensus on gene lists for the diagnosis of myopathies using next-generation sequencing. Eur. J. Hum. Genet. 27, 349-352 (2019)

6. Gorokhova, S. et al. Comparing targeted exome and whole exome approaches for genetic diagnosis of neuromuscular disorders. Appl Transl Genom 7, 26-31 (2015).

7. Tarasov, A., Vilella, A. J., Cuppen, E., Nijman, I. J. \& Prins, P. Sambamba: fast processing of NGS alignment formats. Bioinformatics 31, 2032-2034 (2015).

8. Pedersen, B. S. \& Quinlan, A. R. Mosdepth: quick coverage calculation for genomes and exomes. Bioinformatics 34, 867-868 (2018).

9. Doig, K. D. et al. PathOS: a decision support system for reporting high throughput sequencing of cancers in clinical diagnostic laboratories. Genome Med. 9, 38 (2017).

10. Münz, M. et al. CoverView: a sequence quality evaluation tool for next generation sequencing data. Wellcome Open Res 3, 36 (2018).

\section{Acknowledgements}

We would like to thank the initial users of CovReport at the Laboratory of Molecular Genetics at the Timone Hospital, Marseille (Caroline Lacoste, Véronique Blanc, Christope Pecheux and Amandine Boyer) as well as Henri Pégeot at the Laboratory of Molecular Genetics, Montpellier, France for the valuable comments and suggestions for improvement. We would also like to thank the Genomics\&Bioinformatics Platform at MMG for providing the exome sequencing data for NA12878.

\section{Author contributions}

M.G. wrote the software, participated in the designing of the tool and in writing of the manuscript. M.C., J.M., F.R., N.L., M.B. and M.K. participated in the testing of the tool and commented on the manuscript. S.G. conceived the tool, participated in the designing of the tool and wrote the manuscript. 


\section{Competing interests}

The authors declare no competing interests.

\section{Additional information}

Supplementary information is available for this paper at https://doi.org/10.1038/s41598-020-63079-4.

Correspondence and requests for materials should be addressed to S.G.

Reprints and permissions information is available at www.nature.com/reprints.

Publisher's note Springer Nature remains neutral with regard to jurisdictional claims in published maps and institutional affiliations.

(c) (1) Open Access This article is licensed under a Creative Commons Attribution 4.0 International License, which permits use, sharing, adaptation, distribution and reproduction in any medium or format, as long as you give appropriate credit to the original author(s) and the source, provide a link to the Creative Commons license, and indicate if changes were made. The images or other third party material in this article are included in the article's Creative Commons license, unless indicated otherwise in a credit line to the material. If material is not included in the article's Creative Commons license and your intended use is not permitted by statutory regulation or exceeds the permitted use, you will need to obtain permission directly from the copyright holder. To view a copy of this license, visit http://creativecommons.org/licenses/by/4.0/.

(C) The Author(s) 2020 